\title{
Prescribing rates and characteristics of recipients of tenofovir-containing regimens before and after market entry of tenofovir alafenamide
}

\author{
Anna Hung, PharmD, PhD, MS; Matthew Sinclair, MD; Marion Hemmersbach-Miller, MD, PhD; \\ Daniel Edmonston, MD; and Christina Wyatt, MD, MS
}

\section{What is already known about this subject}

- Tenofovir disoproxil fumarate (TDF), one of the most widely used antiretroviral drugs since its approval in 2001, has been implicated in kidney injury and loss of bone mineral density.

- In clinical trials, tenofovir alafenamide (TAF) demonstrated favorable outcomes related to kidney injury and loss of bone mineral density, but increased lipid levels compared with TDF.

- Whether the favorable short-term endpoints assessed in these clinical trials outweigh any negative lipid effects and lead to favorable longterm outcomes is currently unknown.

\section{What this study adds}

- Approximately 2.5 years after approval, TAF prescribing rates had increased to match TDF prescribing rates in a large academic health system.

- Compared with TDF, TAF was more likely to be newly prescribed to patients with comorbidities, such as chronic kidney disease, as well as hyperlipidemia and cardiovascular disease.

\section{ABSTRACT \\ BACKGROUND: Tenofovir alafenamide (TAF) is a new formulation of tenofovir disoproxil fumarate (TDF) that was approved in 2015. While clinical trial evidence suggests that TAF has more favorable outcomes related to kidney injury and loss of bone mineral density, TAF also leads to higher lipid levels compared with TDF.}

OBJECTIVES: To (a) determine prescribing rates of TDF and TAF among new recipients from 2014 to 2018 in a large academic health system and (b) compare baseline patient characteristics of those newly prescribed TDF versus TAF before and after the approval of TAF in November 2015.

METHODS: Electronic health record data were used to identify new recipients of TDF or TAF from 2014 to 2018 and describe their total monthly TDF and TAF prescriptions by indication. Patient characteristics were compared among new recipients of TDF before November 2015, new recipients of TDF after November 2015, and new recipients of TAF.

RESULTS: Monthly TAF prescribing rates increased to match TDF prescribing rates by April 2018 (82 vs. 88 prescriptions per month).

\author{
Author affiliations \\ Anna Hung, PharmD, PhD, MS, Duke Clinical \\ Research Institute and Department of \\ Population Health Sciences, Duke University, \\ Durham, NC. Matthew Sinclair, MD; Daniel \\ Edmonston, MD; and Christina Wyatt, \\ MD, MS, Duke Clinical Research Institute \\ and Department of Medicine, Division of \\ Nephrology, Duke University, Durham, NC. \\ Marion Hemmersbach-Miller, MD, PhD, Duke \\ Clinical Research Institute and Duke Division \\ of Infectious Diseases, Duke University, \\ Durham, NC.
}

AUTHOR CORRESPONDENCE:

Anna Hung, 919.668.1493,

anna.hung@duke.edu

J Manag Care Spec Pharm 2020;26(12):1582-88

Copyright $\odot 2020$, Academy of Managed Care Pharmacy. All rights reserved.

TAF recipients and new recipients of TDF before November 2015 had similar racial distributions; both of these groups were more likely to be Black compared with new recipients of TDF after November 2015 (55\% and $53 \%$ vs. $37 \% ; P<0.0001)$. TAF recipients also tended to have more comorbidities, including chronic kidney disease ( $7 \%$ vs. $2 \%$ and $2 \%$; $P<0.0001$ ), hepatitis $C$ virus ( $8 \%$ vs. $5 \%$ and $3 \% ; P<0.0001)$, diabetes ( $13 \%$ vs. $5 \%$ and $6 \%$; $P<0.0001$ ), hypertension ( $27 \%$ vs. $13 \%$ and $13 \% ; P<0.0001)$, coronary artery disease $(5 \%$ vs. $3 \%$ and $2 \% ; P<0.0001)$, hyperlipidemia ( $21 \%$ vs. $6 \%$ and $7 \% ; P<0.0001$ ), and 
congestive heart failure ( $3 \%$ vs. $1 \%$ and $1 \% ; P<0.0001)$, compared with both new recipients of TDF before and after November 2015.

CONCLUSIONS: TAF prescribing rates grew substantially in the 2.5 years after FDA approval. TAF is being prescribed more often than TDF in patients with chronic kidney disease and in patients with cardiovascular disease, suggesting that prescribers may be prioritizing the kidney safety profile over the effect on lipids.

Human immunodeficiency virus (HIV) infection is associated with an increased risk of chronic kidney disease. ${ }^{1-4} \mathrm{Teno}^{-}$ fovir disoproxil fumarate (TDF), one of the most widely used antiretroviral drugs since its approval in 2001, has been implicated in kidney injury and loss of bone mineral density., ${ }^{1,5-7}$ In addition to HIV treatment, TDF-containing regimens are approved for HIV pre-exposure prophylaxis (PrEP), HIV postexposure prophylaxis (PEP), and treatment of chronic hepatitis B virus (HBV) or HIV/HBV coinfection.

In November 2015, the U.S. Food and Drug Administration (FDA) approved a new formulation, tenofovir alafenamide (TAF), for use in the treatment of HIV, HBV, and HIV/HBV coinfection. ${ }^{8}$ In clinical trials, TAF led to smaller decreases in estimated glomerular filtration rate, less proteinuria and albuminuria, smaller decreases in bone mineral density in the spine, and increased bone mineral density in treatment-experienced patients switching from a TDFto a TAF-containing regimen. ${ }^{9-19}$ However, increases in low-density lipoprotein and triglycerides were greater in patients treated with TAF than in those treated with TDF. ${ }^{9-}$ ${ }^{19}$ Whether the favorable short-term endpoints assessed in these clinical trials outweigh any negative lipid effects and lead to favorable long-term outcomes is currently unknown. In the midst of this uncertainty, an assessment of clinician-prescribing patterns for TAF and TDF may inform future comparative effectiveness studies.

The primary objective of this study was to determine prescribing rates of TDF- and TAF-containing regimens among new recipients from 2014 to 2018 in the Duke University Health System. The secondary objective was to compare baseline patient characteristics of those newly prescribed TDF- versus TAF-containing regimens before and after November 2015.

\section{Methods}

\section{STUDY POPULATION}

New recipients of TDF or TAF between January 2014 and December 2018 in the Duke University Health System
(DUHS) were identified. DUHS is located in Durham, NC, and consists of 3 acute care hospitals, as well as multiple ambulatory primary care and specialty clinics. DUHS electronic health record data, which contain clinical, billing, and demographic information, were extracted using a webbased tool called the Duke Enterprise Data Unified Content Explorer. ${ }^{20}$ To ensure new use of TDF, a 1-year lookback period was used, and patients with TDF use before 2014 were excluded.

\section{TREATMENT INDICATION FOR EACH PRESCRIPTION}

Total monthly prescriptions for any TDF- or TAF-containing medication (Supplementary Table 1, available in online article) were extracted for the study cohort from January 2014 to December 2018. For each prescription, TDF or TAF was classified as used for HIV treatment if in the year before the prescription there was either an encounter with a relevant diagnosis code for HIV treatment (Supplementary Table 2, available in online article) or the patient had been prescribed at least 3 antiretroviral medications. In accordance with labeling, TDF prescriptions could also be for HIV PrEP or PEP. Thus, if the TDF prescription was not determined to be used for HIV treatment and there was evidence of use in combination with emtricitabine (FTC) for more than 30 days, then the indication was classified as HIV PrEP.

Alternatively, if the patient had been prescribed TDF and FTC for less than 30 days, this prescription was determined to be for HIV PEP (Supplementary Figure 1, available in online article). TAF was not approved by the FDA for use as PrEP during the study period. Use of TDF or TAF for HBV treatment was determined based on the presence of at least 1 encounter with a relevant diagnosis code (Supplementary Table 2, available in online article) in the year before the prescription.

\section{DEMOGRAPHICS AND COMORBIDITIES}

Demographics and comorbidities were measured at first use of TDF or TAF. Comorbidities were defined based on the patient having at least 1 relevant diagnosis or procedure code (Supplementary Table 2, available in online article) in the year before first use of TDF or TAF.

\section{STATISTICAL ANALYSIS}

The number of TDF and TAF prescriptions prescribed every month from January 2014 to December 2018 among new recipients of TDF and TAF was reported. The number of prescriptions was stratified by use for HIV treatment, HIV PrEP, HIV PEP, and/or HBV treatment. Demographics and comorbidities were compared between new recipients of TDF before November 2015 (when TAF was approved), new 


\section{FIGURE 1 Number of TDF and TAF Prescriptions by Month from 2014 to $2018^{\mathrm{a}}$}

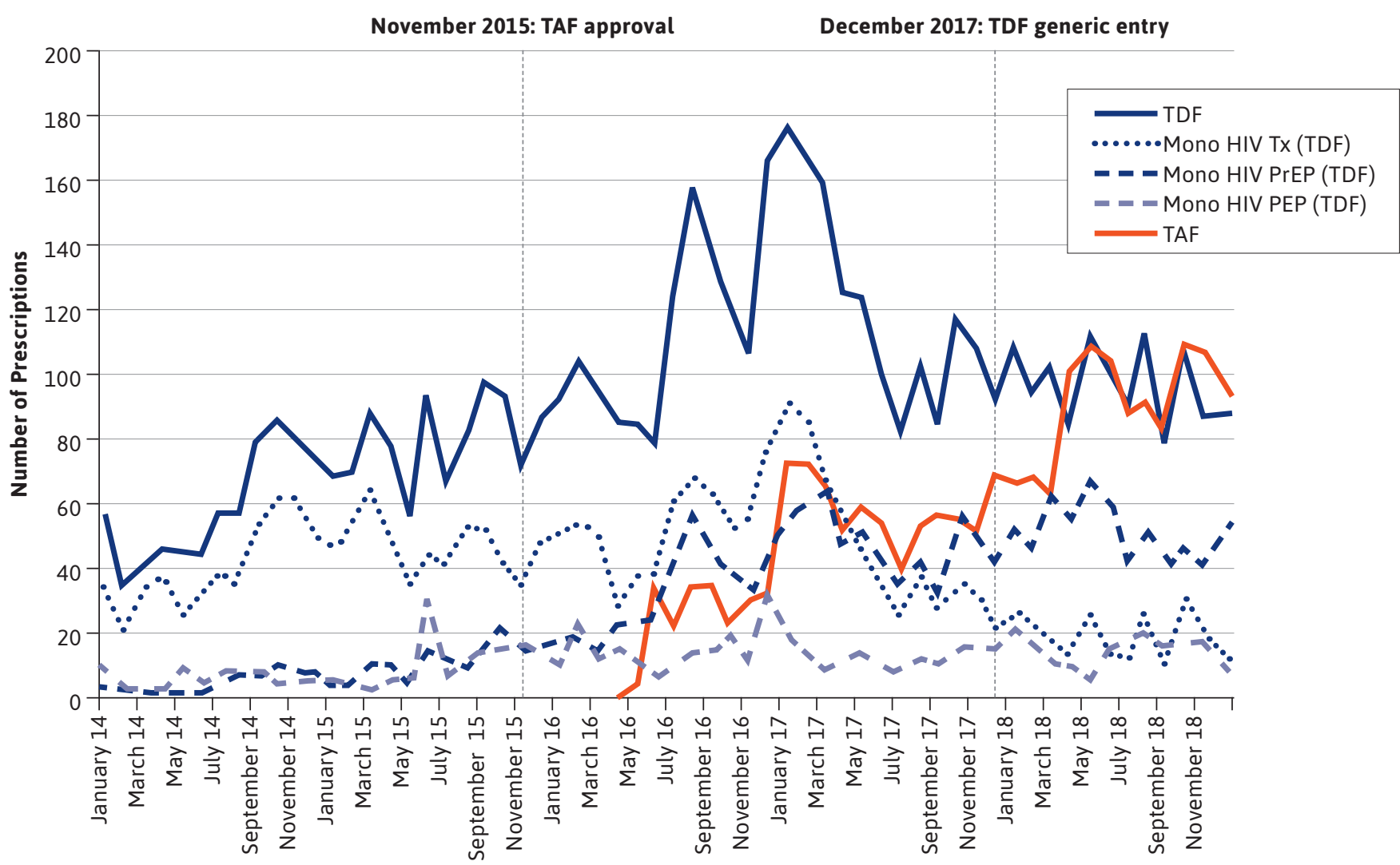

aMono-HBV treatment and cotreatment of HIV and HBV not shown due to small numbers.

$H B V=$ hepatitis $B$ virus; $P E P=$ postexposure prophylaxis; $P r E P=$ pre-exposure prophylaxis; $T A F=$ tenofovir alafenamide; $T D F=$ tenofovir disoproxil fumarate; $T X=$ treatment.

recipients of TDF on or after November 2015, and new recipients of TAF on or after November 2015. Use of TAF before November 2015 was assumed to occur within the context of a clinical trial and was not included in this analysis.

Chi-square tests and Fisher's exact test were used to compare user groups. All tests were 2-sided, and a $P$ value of $<0.05$ was considered statistically significant. All statistical analyses were performed in SAS version 9.4 (SAS Institute, Cary, NC). This study was approved by the Duke University Health System Institutional Review Board.

\section{Results}

We identified 4,238 patients who were prescribed TDF or TAF between 2014 and 2018. Of these, 714 patients had initially been prescribed TDF before 2014 and were thus excluded from analyses. Therefore, our final cohort included 3,524 patients.

\section{MONTHLY PRESCRIBING RATES}

In the study cohort, TDF prescribing increased from 55 prescriptions per month in January 2014 to 88 prescriptions per month in December 2018 (Figure 1). Over the study period, the proportion of TDF prescriptions for HIV treatment decreased (63\% in January 2014 to 15\% in December 2018) as the proportion for HIV PrEP increased substantially (6\% in January 2014 to 67\% in December 2018). The proportion of TDF prescriptions for HIV PEP remained relatively constant (16\% in January 2014 to 11\% in December 2018). Meanwhile, TAF prescriptions emerged in April 2016 (5 months after FDA approval) and increased to levels close to that of TDF by April 2018 (82 prescriptions per month). The majority of TAF prescriptions were written for HIV treatment alone. For TDF 


\section{TABLE 1 Characteristics of New Recipients of TDF vs. TAF}

\begin{tabular}{|c|c|c|c|c|}
\hline \multirow[b]{2}{*}{ Demographics, n (\%) } & \multirow{2}{*}{\begin{tabular}{|c|} 
Before \\
November 1,2015 \\
$\begin{array}{c}\text { New Recipient of } \\
\text { TDF }(n=880)\end{array}$ \\
\end{tabular}} & \multicolumn{2}{|c|}{$\begin{array}{c}\text { On or After } \\
\text { November 1, } 2015\end{array}$} & \multirow[b]{2}{*}{$P$ Value } \\
\hline & & $\begin{array}{l}\text { New Recipient of } \\
\operatorname{TDF}(n=1,778)\end{array}$ & $\begin{array}{c}\text { New User of TAF } \\
(n=866)\end{array}$ & \\
\hline \multicolumn{5}{|l|}{ Age, years } \\
\hline $0-18$ & $34 \quad(4)$ & 115 & $(0.5)$ & \multirow{5}{*}{$<0.0001$} \\
\hline $19-30$ & $223 \quad(25)$ & $674 \quad(38)$ & $112 \quad(13)$ & \\
\hline $30-45$ & $297 \quad(34)$ & $511 \quad(29)$ & $222 \quad(26)$ & \\
\hline $45-64$ & $298 \quad(34)$ & $427 \quad(24)$ & $435 \quad(50)$ & \\
\hline $65+$ & (3) & (3) & (11) & \\
\hline \multicolumn{5}{|l|}{ Sex } \\
\hline Female & $324 \quad(37)$ & $572 \quad(32)$ & $268 \quad(31)$ & 0.0183 \\
\hline \multicolumn{5}{|l|}{ Race } \\
\hline White & $277 \quad(31)$ & (43) & $257 \quad(30)$ & \multirow{4}{*}{$<0.0001$} \\
\hline Black & $469 \quad(53)$ & $(37)$ & $478 \quad(55)$ & \\
\hline Other & $111 \quad(13)$ & $(15)$ & $107 \quad(12)$ & \\
\hline Unknown & $23 \quad(3)$ & $(4)$ & (3) & \\
\hline \multicolumn{5}{|l|}{ Ethnicity } \\
\hline Hispanic & 56 & 140 & (6) & \multirow{3}{*}{0.0752} \\
\hline Not Hispanic & $793 \quad(90)$ & $1,557 \quad(88)$ & $788 \quad(91)$ & \\
\hline Unknown & 31 & $(5)$ & (3) & \\
\hline \multicolumn{5}{|l|}{ Marital status } \\
\hline Married & $144 \quad(16)$ & (19) & $214 \quad(25)$ & \multirow{4}{*}{$<0.0001$} \\
\hline Single & $561 \quad(64)$ & 1,139 & $(56)$ & \\
\hline Other & $94 \quad(11)$ & 136 & $99 \quad(11)$ & \\
\hline Unknown & $81 \quad(9)$ & 172 & (8) & \\
\hline
\end{tabular}

continued on next page

and TAF prescribing, use for treatment of HBV mono-infection was low (median of $8 \%$ of TDF prescriptions and $12 \%$ of TAF prescriptions per month).

\section{PATIENT DEMOGRAPHICS AND COMORBIDITIES}

TAF recipients tended to be older $(66 \%$ of TAF recipients were aged 45 years and above vs. $27 \%$ and $37 \%$ of new recipients of TDF before and after November 2015, respectively; $\mathrm{P}<0.0001$ ) and were more likely to be male $(69 \%$ vs. $68 \%$ and $63 \% ; \mathrm{P}=0.0183$ ) and married $(25 \%$ vs. $19 \%$ and $16 \% ; \mathrm{P}<0.0001)$ than new recipients of TDF before or after November 2015 (Table 1). TAF recipients and new recipients of TDF before November 2015 had similar racial distributions; both of these groups were more likely to be Black compared with new recipients of TDF after November 2015 (55\% and 53\% vs. 37\%; $\mathrm{P}<0.0001$ ).

TAF recipients were more likely being treated for HIV treatment alone $(82 \%$ vs. $51 \%$ or $21 \%$; $\mathrm{P}<0.0001)$, cotreatment of HIV and HBV (4\% vs. $1 \%$ or $0.3 \% ; \mathrm{P}<0.0001)$, and $\mathrm{HBV}$ treatment alone $(12 \%$ vs. $5 \%$ or
7\%; $\mathrm{P}<0.0001)$ compared with TDF recipients starting before or after November 2015. TDF recipients starting after November 2015 were more likely to use TDF for HIV PrEP (37\% vs. $14 \%$; $P<0.0001)$ or PEP $(27 \%$ vs. $18 \%$; $\mathrm{P}<0.0001)$ compared with TDF recipients starting before November 2015.

New recipients of TAF also tended to have more comorbidities, including chronic kidney disease (7\% vs. $2 \%$ and $2 \%$; $P<0.0001$ ), hepatitis $C$ virus ( $8 \%$ vs. $5 \%$ and $3 \% ; \mathrm{P}<0.0001)$, diabetes $(13 \%$ vs. $5 \%$ and $6 \%$; $P<0.0001)$, hypertension (27\% vs. $13 \%$ and $13 \% ; P<0.0001)$, coronary artery disease (5\% vs. $3 \%$ and $2 \% ; \quad P<0.0001)$, hyperlipidemia (21\% vs. $6 \%$ and $7 \% ; \mathrm{P}<0.0001$ ), and congestive heart failure (3\% vs. $1 \%$ and $1 \% ; \mathrm{P}<0.0001$ ), compared with new recipients of TDF before and after November 2015. Documented diagnosis of osteoporosis was rare, and although it was more common in new TAF recipients, there was no statistically significant difference between the groups ( $1 \%$ vs. $0.2 \%$ and $0.5 \%$; $P=0.2204)$.

\section{Discussion}

In this study, TAF prescribing rates increased to levels similar to that of TDF prescribing rates approximately 2.5 years after FDA approval. To our knowledge, this is the first study to examine TDF and TAF monthly prescribing patterns in a U.S. health system. Although a generic version of the singleagent TDF drug product was made available in December 2017, ${ }^{21}$ no substantial increase in TDF prescribing rates was seen after December 2017.

This study found prescribing differences by race and comorbidity. After TAF entered the market, we found that prescribers were more likely to prescribe TAF than TDF in Black populations. This may be due to clinicians prescribing TAF to patients with comorbidities such as chronic 


\begin{tabular}{|c|c|c|c|c|}
\hline \multirow[b]{2}{*}{ Demographics, n (\%) } & \multirow{2}{*}{\begin{tabular}{|c|} 
Before \\
November 1, 2015 \\
$\begin{array}{c}\text { New Recipient of } \\
\text { TDF }(n=880)\end{array}$ \\
\end{tabular}} & \multicolumn{2}{|c|}{$\begin{array}{c}\text { On or After } \\
\text { November 1, } 2015\end{array}$} & \multirow[b]{2}{*}{$P$ Value } \\
\hline & & $\begin{array}{l}\text { New Recipient of } \\
\text { TDF }(n=1,778)\end{array}$ & $\begin{array}{c}\text { New User of TAF } \\
(n=866)\end{array}$ & \\
\hline \multicolumn{5}{|l|}{ Treatment indication, $\mathrm{n}(\%)$} \\
\hline HIV Tx only & 449 & $(21)$ & $(82)$ & \multirow{5}{*}{$<0.0001$} \\
\hline Co-Tx of HIV and HBV & $(1)$ & $(0.3)$ & (4) & \\
\hline HBV Tx only & 44 & 119 & $(12)$ & \\
\hline HIV PrEP only & $124 \quad(14)$ & $661 \quad(37)$ & N/A & \\
\hline HIV PEP only & 156 & $(27)$ & N/A & \\
\hline \multicolumn{5}{|l|}{ Comorbidities, $n(\%)$} \\
\hline Hepatitis C virus & (5) & (3) & (8) & \multirow{4}{*}{$<0.0001$} \\
\hline Diabetes & $46 \quad(5)$ & $(6)$ & $115 \quad(13)$ & \\
\hline Hypertension & $116 \quad(13)$ & $239 \quad(13)$ & $237 \quad(27)$ & \\
\hline Chronic kidney disease & 19 & $(2)$ & (7) & \\
\hline Osteoporosis & $2 \quad(0.2)$ & $(0.5)$ & (1) & $0.2204^{a}$ \\
\hline Coronary artery disease & 22 & $(2)$ & (5) & \multirow{2}{*}{$<0.0001$} \\
\hline Hyperlipidemia & $(6)$ & 122 & $182 \quad(21)$ & \\
\hline Congestive heart failure & 11 & (1) & (3) & 0.0059 \\
\hline \multicolumn{5}{|c|}{$\begin{array}{l}\text { aFisher's exact test since } 33 \% \text { of cells have expected counts of }<5 \text {. } \\
\text { CO-TX=cotreatment; } H B V=\text { hepatitis } B \text { virus; } N / A=\text { not applicable; } P E P=\text { postexposure prophylaxis; } \\
\text { PrEP= pre-exposure prophylaxis; } T A F=\text { tenofovir alafenamide; } T D F=\text { tenofovir disoproxil fumarate; } \\
T X=\text { treatment. }\end{array}$} \\
\hline
\end{tabular}

kidney disease that are more prevalent in Black populations.

Furthermore, this study found that new recipients of TAF were more likely to have comorbidities than new recipients of TDF. Because the extensive literature that implicates TDF use in kidney injury and loss of bone mineral density, ${ }^{1,5-7}$ it is not surprising to see higher prescribing rates of TAF compared with TDF in populations with chronic kidney disease and osteoporosis. However, given that TAF has been shown to worsen lipid levels when compared with $\mathrm{TDF}^{17,18}$ it is notable that TAF is being prescribed more often than TDF in patients with hyperlipidemia and cardiovascular disease.

Interestingly, TAF was more likely to be prescribed in populations with comorbidities compared with TDF exist only with TAF and not TDF. Future research should examine prescribing rates of individual TDF and TAF drug products to attribute increases or decreases to specific products.

\section{LIMITATIONS}

This study has some limitations to consider. First, TDF and TAF prescribing patterns were examined among those newly started on TDF or TAF between 2014 and 2018 in a single health system. We limited the cohort to new recipients of TDF or TAF between 2014 and 2018 because not all data regarding patient characteristics before 2014 were available; thus, TDF prescribing trends for those who started on TDF before 2014 were not included.

Furthermore, a single health system was examined and whether similar prescribing patterns exist in other health systems is unknown. Nonetheless, the health system includes inpatient and ambulatory care clinics in an academic medical center and 2 large community hospitals, increasing the generalizability of our findings.

Second, comorbidities were classified based on electronic health record-based diagnosis and procedure codes and may have been under- or overreported within the health records. Future studies could use electronic health records and administrative claims data, which may allow for more accurate classification of comorbidities because administrative claims are used for billing.

Third, this study did not examine use of TAF for PrEP because TAF was not approved for PrEP until after our study period. Future studies should examine to what degree TAF versus TDF is being used for PrEP, especially considering recent evidence that, compared with TDF/FTC, TAF/FTC is not cost-effective for PrEP unless there are substantial discounts to its current list price. ${ }^{22}$ Moreover, a generic version of the combination 
TDF/FTC drug product was recently made available, ${ }^{23}$ so it will be interesting to see whether there will be an increase in TDF prescribing.

\section{Conclusions}

This study examined TDF and TAF prescribing patterns and found that TAF prescribing rates had increased to match TDF prescribing rates approximately 2.5 years after FDA approval. Compared with TDF, TAF was more likely to be newly prescribed to patients with comorbidities, including chronic kidney disease, as well as hyperlipidemia and cardiovascular disease. These results suggest that clinicians are prescribing TAF for a patient population broader than that for TDF to include populations with comorbidities and that prescribers may be prioritizing data in support of an improved kidney safety profile over data suggesting a less favorable effect of TAF on lipids.

\section{DISCLOSURES}

This work was supported by the Duke Clinical Research Institute Executive Director's Pathway for Supplemental Funding. The research team received additional support from the National Institute of Diabetes, Digestive, and Kidney Disease R01DK112258 and P01DK056492 (CW) and from the National Institute of Allergy and Infectious Diseases 5T32AI100851 (MHM). The content is solely the responsibility of the authors and does not necessarily represent the official views of the National Institutes of Health. Hung reports past employment by Blue Cross Blue Shield Association and CVS Health and a grant from Pharmaceutical Research and Manufacturers of America (PhRMA), unrelated to this work. The other authors have nothing to disclose.

This work was accepted as a poster presentation for the AMCP Nexus 2020 Virtual, October 19-23, 2020.

\section{ACKNOWLEDGMENTS}

The authors thank Michael Chrestensen, Caitlin Fisher, Pamela Barth, and Todd Hale for data support.

\section{REFERENCES}

1. Wyatt CM. Kidney disease and HIV infection. Top Antivir Med. 2017;25(1):13-16.

2. Rosenberg AZ, Naicker S, Winkler CA, Kopp JB. HIV-associated nephropathies: epidemiology, pathology, mechanisms and treatment. Nat Rev Nephrol. 2015; 11(3):150-60.

3. Cohen SD, Kopp JB, Kimmel PL. Kidney diseases associated with human immunodeficiency virus infection. N Engl J Med. 2017;377(24):2363-74.

4. Swanepoel CR, Atta MG, D'Agati VD, et al. Kidney disease in the setting of HIV infection: conclusions from a Kidney Disease: Improving Global Outcomes (KDIGO) Controversies Conference. Kidney Int. 2018;93(3):545-59.

5. Tourret J, Deray G, Isnard-Bagnis C. Tenofovir effect on the kidneys of HIVinfected patients: a double-edged sword? J Am Soc Nephrol. 2013;24(10):1519-27.

6. Cooper, RD, Wiebe N, Smith N, Keiser P, Naicker S, Tonelli M. Systematic review and meta-analysis: renal safety of tenofovir disoproxil fumarate in HIV-infected patients. Clin Infect Dis. 2010;51(5):496-505.

7. Mocroft A, Lundgren JD, Ross M, et al. Cumulative and current exposure to potentially nephrotoxic antiretrovirals and development of chronic kidney disease in HIV-positive individuals with a normal baseline estimated glomerular filtration rate: a prospective international cohort study. Lancet HIV. 2016;3(1):e23-32.

8. U.S. Food and Drug Administration. Drugs@FDA: FDA-approved drugs.

Accessed November 2, 2020. https:// www.accessdata.fda.gov/scripts/cder/ daf/index.cfm

9. Ray AS, Fordyce MW, Hitchcock MJ. Tenofovir alafenamide: a novel prodrug of tenofovir for the treatment of human immunodeficiency virus. Antiviral Res. 2016;125:63-70.
10. Gupta SK, Pozniak A, Arribas J, et al. Subjects with renal impairment switching from tenofovir disoproxil fumarate to tenofovir alafenamide have improved renal and bone safety through 48 weeks [abstract]. Poster presented at: 8th IAS Conference on HIV Pathogenesis, Treatment and Prevention; July 18-22, 2015: Vancouver, Canada. Accessed November 2, 2020. https://www.natap. org/2015/IAS/IAS 23.htm

11. Mills A, Arribas JR, Andrade-Villanueva J, et al. Switching from tenofovir disoproxil fumarate to tenofovir alafenamide in antiretroviral regimens for virologically suppressed adults with HIV-1 infection: a randomised, active-controlled, multicentre, open-label, phase 3, non-inferiority study. Lancet Infect Dis. 2015;16(1):43-52.

12. Mills A, Crofoot Jr G, McDonald C, et al. Tenofovir alafenamide versus tenofovir disoproxil fumarate in the first protease inhibitor-based single-tablet regimen for initial HIV-1 therapy: a randomized phase 2 study. J Acquir Immune Defic Syndr. 2015;69(4):439-45.

13. Pozniak A, Arribas JR, Gupta SK, et al. Safety of tenofovir alafenamide in renal impairment [abstract 795]. Poster presented at: Conference on Retroviruses and Opportunistic Infections (CROI); February 23-26, 2015; Seattle, WA. Accessed November 2, 2020. https:// www.croiconference.org/abstract/ safety-tenofovir-alafenamide-renalimpairment/\#: :text=Conclusions \%3A\%20These\%2024\%20week\%20 data,and\%20with\%20reductions\%20 in\%20proteinuria

14. Sax PE, Wohl D, Yin MT, et al. Tenofovir alafenamide versus tenofovir disoproxil fumarate, coformulated with elvitegravir, cobicistat, and emtricitabine, for initial treatment of HIV-1 infection: two randomised, double-blind, phase 3, non-inferiority trials. Lancet. 2015; 385(9987):2606-15.

15. Sax PE, Zolopa A, Brar I, et al. Tenofovir alafenamide vs. tenofovir disoproxil fumarate in single tablet regimens for initial HIV-1 therapy: a randomized phase 2 study. J Acquir Immune Defic Syndr. 2014;67(1):52-58. 
16. Grund B, Peng G, Gibert CL, et al. Continuous antiretroviral therapy decreases bone mineral density. AIDS. 2009;23(12):1519-29.

17. Kauppinen KJ, Kivelä P, Sutinen J. Switching from tenofovir disoproxil fumarate to tenofovir alafenamide significantly worsens the lipid profile in a realworld setting. AIDS Patient Care STDS. 2019;33(12):500-06.

18. Cid-Silva P, Fernández-Bargiela N, Margusino-Framiñán L, et al. Treatment with tenofovir alafenamide fumarate worsens the lipid profile of HIV-infected patients versus treatment with tenofovir disoproxil fumarate, each coformulated with elvitegravir, cobicistat, and emtricitabine. Basic Clin Pharmacol Toxicol. 2019;124(4):479-90.
19. Wang H, Lu X, Yang X, Xu N. The efficacy and safety of tenofovir alafenamide versus tenofovir disoproxil fumarate in antiretroviral regimens for HIV-1 therapy: meta-analysis. Medicine (Baltimore). 2016; 95(41):e5146.

20. Horvath MM, Winfield S, Evans S, Slopek S, Shang H, Ferranti J. The DEDUCE Guided Query tool: providing simplified access to clinical data for research and quality improvement. J Biomed Inform. 2011;44(2):266-76.

21. Hoffman M. FDA grants final approval to tenofovir disoproxil fumarate generic. January 29, 2018. Accessed November 2, 2020. https://www.mdmag.com/medicalnews/fda-grants-final-approval-to-tenofovir-disoproxil-fumarate-generic
22. Walensky RP, Horn T, McCann NC, Freedberg KA, Paltiel AD. Comparative pricing of branded tenofovir alafenamideemtricitabine relative to generic tenofovir disoproxil fumarate-emtricitabine for HIV preexposure prophylaxis: a costeffectiveness analysis. Ann Intern Med. 2020;172(9):583-90.

23. Highleyman, L. First generic Truvada now available in the United States. October 2, 2020. Accessed November 10, 2020. https://www.poz.com/article/firstgeneric-truvada-now-available-united$\underline{\text { states }}$ 